\title{
Does acupuncture have any effect on obstructed defecation syndrome?
}

\author{
(1) SHUQING DING ${ }^{1}$, (1) YIJIANG DING², (1) LINGLING WANG ${ }^{3}$, (1) HUIFENG ZHOU², (1) XUN JIN³ \\ 1Pelvic Floor Medicine Committee of World Federation of Chinese Medicine Societies, Nanjing, China \\ ${ }^{2}$ National Center of Colorectal Diseases of Chinese Medicine, Affiliated Hospital of Nanjing University of Chinese Medicine, Nanjing, China \\ ${ }^{3}$ Nanjing University of Chinese Medicine, Nanjing, China
}

\begin{abstract}
Objective: Obstructed defecation syndrome (ODS) is a group of symptoms that are mainly caused by pelvic floor dysfunction concerning bowel symptoms. It is challenging in clinical practice. Acupuncture has advantages in the treatment of complex symptoms because of its multitarget and bi-directional regulation of the human body. Since, 2,500 years ago, acupuncture has been applied empirically to the treatment of constipation. Does acupuncture have any effect on ODS?
\end{abstract}

By showing the clinical thoughts, methods, and achievements of acupuncture series in ODS in recent ten years by the author's team, and two scientific papers published in English on acupuncture treatment of chronic intractable constipation and stress urinary incontinence, it is proved that the core scheme of acupuncture treatment of ODS is flexible.

In order to raise awareness of the therapeutic effect of acupuncture in ODS, it can be integrated into existing practice to get opportunities for multi-disciplinary cooperation and further research and development.

Materials and Methods: By summarizing the diagnosis and treatment of ODS, the first-line selection of pelvic floor rehabilitation and the minimally invasive sacral neuromodulation were reviewed, and it was pointed out that there was a gap between the high demand of patients and the expectation of cost-effectiveness. Then, on the basis of modern eastern and western medical achievements, the holistic concept was introduced into the treatment of pelvic floor dysfunction, and an acupuncture scheme suitable for ODS was proposed.

Results: Acupuncture is based on the idea of improving the patients' central nervous system, autonomic nervous system, and intestinal nervous system, and is effective in treating ODS. The acupoints were set in two groups when the patient in a supine position, which includes ST 25 (Tian Shu), SP 15 (Da Heng), SP 14 (Fu Jie), CV 6 (Qi Hai), CV 4 (Guan Yuan), ST 36 (Zu San Li), ST 37 (Shang Ju Xu); When a patient is in the prone position, it includes BL 20 (Pi Shu), BL 23 (Shen Shu), BL 25 (Da Chang Shu), BL 33 (Zhong Liso), BL 34 (Xia Liao), and GV 20 (Bai Hui). The key was the technique of deep needling of the ST 25 (Tian Shu) and deep needling of the BL 33 (Zhong Liso) \& BL 34 (Xia Liao). It needs 2-15 $\mathrm{Hz}$ sparse-dense wave electrical stimulation, 30 minutes each time, a total of 20 times, which was a scheme that could achieve satisfactory short-term and long-term effects.

Conclusion: At present, clinical and basic experimental studies have proved that acupuncture plays a role in treating ODS in a multi-target way. This is a very promising research direction of pelvic floor integrated medicine.

In the future, further study on optimizing the protocol and meeting the patient's gap individually and cost-effectively.

Keywords: Acupuncture; obstructive defecation syndrome; pelvic floor symptoms; constipation; protocol; electronic stimulation; sacral nerve modulation; pelvic floor rehabilitation

Address for Correspondence: Shuqing Ding, Pelvic Floor Medicine Committee of World Federation of Chinese Medicine Societies, Nanjing, China E-mail: njgczx@gmail.com ORCID ID: orcid.org/0000-0002-5875-8629

Received: 16 November 2020 Accepted: 12 February 2021

${ }^{\circ}$ Copyright 2021 by the International Society for Pelviperineology / Pelviperineology published by Galenos Publishing House. 


\section{INTRODUCTION}

Obstructed defecation syndrome (ODS) is a type of constipation, which is a kind of pelvic floor dysfunction disease concerning bowel symptoms, characterized by fragmented stools, overstraining at defecation, sense of incomplete evacuation, tenesmus, urgency, pelvic heaviness, and self-digitation. ${ }^{1}$

A pelvic floor is a functional unit connecting the bladder, uterus, rectum, and other organs through muscles and ligaments, which is controlled by the same nervous system at different levels. The functions of any organs in the pelvic floor are balanced with each other, rather than existing alone. In addition to the above-mentioned defecation symptoms, patients may also have overlapping urination, pelvic floor relaxation, and sexual symptoms.

Patients usually cannot get satisfactory results from general lifestyle changes, fiber diet, exercise, and laxatives. Some patients may even become addicted to taking laxatives, suppository, or rectal irrigation. ODS greatly affects patients' quality of life, physically and mentally.

Western medicine doctors may recommend patients to physiotherapists for pelvic floor biofeedback therapy, which is the first choice recognized by gastroenterology, ${ }^{2}$ and colorectal specialists. ${ }^{3}$ However, patients often quit behavioural training from frustration and other reasons.

Some selected patients may benefit from sacral neuromodulation (SNM), a minimally invasive surgical technique, with a total success rate of $73 \%$. It is promising but still not well accepted worldwide mainly due to the cost-effectiveness and complications from the device and operation itself. ${ }^{4}$

In 2008, the author published the preliminary research results of acupuncture treatment in ODS in China and conducted a series of studies later, ${ }^{5-7}$ which showed that acupuncture in ODS had advantages combining with the above-mentioned methods. ${ }^{8}$

It is hoped that through further multidisciplinary cooperation and multi-centre research, acupuncture will be applied and popularized effectively in pelvic floor disorders.

\section{Obstructed defecation syndrome (ODS)}

\section{Symptoms and pathophysiology}

ODS is characterized by fragmented stools, overstraining at defecation, a sense of incomplete evacuation, tenesmus, urgency, pelvic heaviness, and self-digitation. ${ }^{1}$

ODS is frequently encountered in colorectal, gastroenterology, or gynaecology clinics. According to the Rome II Criteria of functional gastrointestinal disorders, the incidence of chronic functional constipation is $6.07 \%$ to $11.5 \%$. In China's general population, the incidence is double in aged female patients. Among these, nearly $60 \%$ of patients with constipation suffer from ODS. ${ }^{8}$

Based on the pelvic floor integral theory of Petros and Ulmsten put forward for the first time in 1990, the pelvic floor is regarded as a functional unit connecting the bladder, uterus, rectum, and other organs through muscles and ligaments, which is controlled by the same nervous system at different levels. The functions of any organs in the pelvic floor are balanced with each other, rather than exist alone. ${ }^{9}$ So in addition to the above-mentioned defecation symptoms, ODS patients may also have overlapping urination, pelvic floor organ prolapse, and sexual symptoms.

Other related pathophysiological factors also involved the pelvic floor dysfunction, including the muscle system (puborectalis muscle and/or the internal anal sphincter can't relax during defecation), peripheral nervous system (sacral parasympathetic nerve and/or pudendal nerve neuropathy), ${ }^{10,11}$ central nervous system (Parkinson's disease), ${ }^{12}$ and psychological or behavioural issues, ${ }^{13}$ etc.

\section{MATERIALS AND METHODS}

\section{Diagnosis and evaluation}

ODS is a challenging disease. A well-trained multidisciplinary team is needed in the pelvic floor center for its comprehensive evaluation. The diagnosis of ODS is based on a careful collection of patients' clinical history. The severity of symptoms may be objectively evaluated using a validated score. ${ }^{14}$ After testing, ODS is defined as three major subtypes of constipation, which are usually identified as normal colonic transit, slow transit, and functional defecation or not. Digital rectal examination is a useful bedside tool for evaluating functional defecation, which is used to identify dyssynergia and rectocele and is convenient to select patients for further confirmatory physiology testing, ${ }^{15}$ including anorectal manometry, transanal or transperineal dynamic ultrasound, ${ }^{16}$ magnetic resonance imaging defecography, ${ }^{17}$ and psychological evaluation which may offer the morphological and functional abnormal evidence of the patients. ${ }^{18}$

Then ODS has subsets of dyssynergic defecation, pelvic floor relaxation, and mixed presentations. Dyssynergic defecation is most often associated with an inability to coordinate abdominal, rectoanal, and pelvic floor muscles during defecation. On rectal examination, the patient may have a high resting tone, and on bear down descent may be poor or paradoxical. Pelvic floor relaxation patients may have a normal or lower resting tone, and on bear down perineum descent great, may manifest 
rectocele, intussusception even pelvic organ prolapse of rectum, intussusception, uterus or bladder. The difference in rectal sensation (hyper- or hypo-) and compliance (hyper- or hypo-) of patients also participate in the above-mentioned different subtypes, thus enriching the details of ODS.

Patients suffering from severe chronic defecation symptoms often represent an acquired behavioural disorder and may have potential psychological issues. In our 90 cases research on chronic functional constipation, 35 (38.9\%) cases had the problem, of which 14 cases had mild to moderate depression while 21 cases had severe depression and anxiety; almost all the patients had sleeping issue. Additionally, patients complain of fatigue, weakness, some female patients may have menstruation shortage or weight loss due to endocrine or nutrition dysfunction. ${ }^{8}$ Although not directly life-threatening, this psychosomatic disease adversely affects a patient's social and personal life as 4 "D", an abbreviation of discomfort, depression, dollar costs, and drug toxicity.

\section{Pelvic floor rehabilitation}

In view of the complexity of etiology, pathology, and overlapping symptoms, a multidisciplinary approach seems to be the key, ${ }^{1}$ which requires a holistic method to fix the functional balance, namely pelvic floor rehabilitation.

Basically, the ODS patients cannot get satisfactory results from the management for constipation, such as changing lifestyle, fiber diet, exercise, laxatives, etc., And some patients may even take more laxatives, suppository, or rectal irrigation for temporary relief. It greatly affects patients' quality of life physically and mentally.

Being safe and non-invasive, pelvic floor biofeedback therapy has been considered as the first choice on ODS for rehabilitation in western medicine, recommended by international guidelines on gastroenterology, ${ }^{2}$ and colorectal. ${ }^{3}$ This kind of retraining from maladaptive behaviour can improve the defecation effort by learning, and help patients to cultivate a better understanding of pelvic floor muscle self-control and brain-gut reflex reconstruction.

A successful biofeedback therapy requires proper cognitive preparation, guidance, and instruction before using the instrument. The success of biofeedback depends on patient selection, a well-trained therapist, and treatment compliance. Biofeedback effectively relieves symptoms of defecatory disorders in $69 \%$ of affected adults. ${ }^{19}$ Therefore, if patients have a mental disability or have severe anxiety and depression, they may have no ability or motivation to fulfil the repeated trial and error training, or if they cannot get the encouragement from a well-trained therapist, they may easily quit from the frustrated behavioural treatment. ${ }^{20}$

\section{Sacral nerve modulation}

If the patients are dissatisfied with non-surgical treatment, some selected patients may benefit from the sacral nerve modulation (SNM), it has shown great potential in treating pelvic floor dysfunction in recent decades. ${ }^{21}$ This minimally invasive procedure includes two stages. Initially, an electrode is implanted into the posterior sacral foramen (mostly the right S3) to stimulate the sacral nerve roots; a temporary electrode lead is connected to a portable battery unit outside the body. After $2-3$ weeks of testing, if symptoms are improved by more than $50 \%$, the second stage operation is considered feasible and this is replaced by the implanted battery for permanent stimulation.

A systematic review, ${ }^{21}$ assessed the therapeutic effects on chronic constipation. There are 13 studies using SNM in chronic constipation all over the world, and the success rate in the first stage is $42 \%-100 \%$. then $87 \%$ of patients improved after the permanent implantation, and the average follow-up time was 28 months. Another systematic review showed that the total "success rate" of patients who received permanent implantation was $73 \%$, and the removal rate of instruments was about $12 \%{ }^{4}$ The balance of risk versus benefit must be fully understood by patients and clinicians, and realistic expectations set out before treatment. ${ }^{4}$

It is promising but still not well accepted worldwide mainly due to the cost-effectiveness and complications from the device and operation itself as well.

On the basis of ODS clinical research and management update, the multidisciplinary pelvic floor team of Nanjing University of Traditional Chinese Medicine and National Center of Integrated Colorectal Surgery started to carry out acupuncture research in the "sacral nerve modulation era" in 2005.

Most of the original works were published in Chinese. This is the first original paper on teamwork and achievement published in English since then. The authors will exhibit a set of acupuncture protocols based on clinical trials and research evidence or animal studies finding, combining with the authors' expertise to meet the gap of patients' needs cost-effectively.

\section{Acupuncture for ODS}

Acupuncture based on traditional Chinese medicine treats patients as a whole. The first record for constipation was from "Inner Canon of Yellow Emperor (Huangdi Neijing)" around 2,500 years ago. It has been empirically practiced in China for several millennia, and the technique treating constipation is now being 
increasingly accepted by practitioners and patients worldwide, including the United States. ${ }^{22,23}$

Acupuncture has a bidirectional holistic and physiologic limit modulation effect in treatment. Bidirectional modulation, namely, an unbalanced (hyperactive or hypoactive) pathophysiological state can be normalized by acupuncture based on the patient physique individually, it is not a purely excitatory or suppression process. Holistic modulation means acupuncture has a multi-target and multi-system effect through meridians by adjusting Yang et al. ${ }^{24}$ It is an option for complex pathological mechanism diseases like chronic constipation.

The goal of the acupuncture program is to regulate the whole pelvic floor muscle and nerve function from the philosophy of Chinese medicine and Western medicine.

\section{RESULTS}

Acupoints selection ${ }^{25}$

The nervous system is composed of the central nervous system (CNS), autonomic nervous system (ANS) (sympathetic nervous system, and parasympathetic nervous systems), and enteric nervous system (ENS). The selection of acupoints in the sacrum, lower abdomen, back, and the head is based on this design concept.

The first group set when the patient in a supine position, which includes ST 25 (Tian Shu), SP 15 (Da Heng), SP 14 (Fu Jie), CV 6 (Qi Hai), CV 4 (Guan Yuan), ST 36 (Zu San Li), ST 37 (Shang Ju Xu); The second group set when patient in the prone position, which includes BL 20 (Pi Shu), BL 23 (Shen Shu), BL 25 (Da Chang Shu), BL 33 (Zhong Liao), BL 34 (Xia Liao) and GV 20 (Bai Hui).

Figure 1 shows the appearance of acupoints and needles penetration.

The most important acupoints are ST 25 (Tian Shu), BL 33 (Zhong Liao) \& BL 34 (Xia Liao), and GV 20 (Bai Hui) which respectively represent the regulation of ENS, ANS, and CNS.

\section{Acupoint ST 25 deep needling technique ${ }^{26}$}

Patients take the supine position, the acupoints ST 25 (Tian Shu) is located two inches $(50 \mathrm{~mm}$ ) away from the navel, use needles (size $75 \times 0.30 \mathrm{~mm}$ ) slowly penetrate $40-50 \mathrm{~mm}$ vertically indepth, according to the thickness of the patient abdominal wall until the needle tip reaches the peritoneal layer.

During the process of needle insertion, the operator feels heavy, with a little resistance, just like the feeling of fish biting the hook, and then continues to deepen the needle. When there is a significant breakthrough under the needle tip, the patient may feel pulled and then stop inserting the needle.

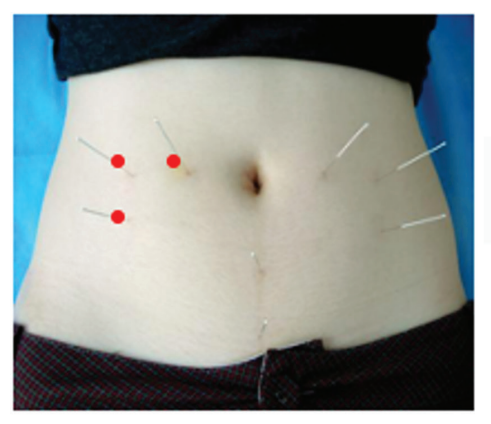

\section{Abdominal Points for ENS}

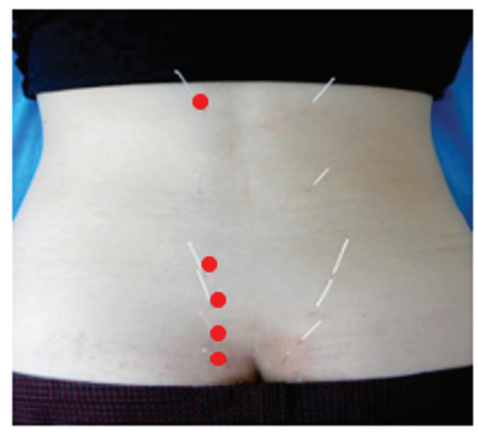

Back Points for ANS

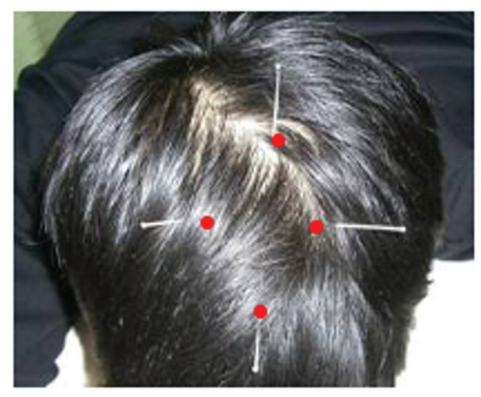

Head Points for CNS

Figure 1. Appearance of needles in patients

ENS: Enteric nervous system, ANS: Autonomic nervous system, CNS: Central nervous system

Compared with the shallow needle (the needle tip only enters the fascia layer, but does not penetrate the peritoneum), the deep needle technique has a faster response.

\section{Acupoints BL33\& BL34 deep needling technique ${ }^{27}$}

Patients take the prone position, the acupoints BL 34 (Xia Liao) and BL 33 (Zhong Liao) are located in the $4^{\text {th }}$ and $3^{\text {rd }}$ sacral foramen, use needles (size $75 \times 0.30 \mathrm{~mm} / 0.40 \mathrm{~mm}$ or $100 \times 0.30$ $\mathrm{mm} / 0.40 \mathrm{~mm}$ ) slowly penetrate $75-100 \mathrm{~mm}$ into the anterior sacral foramen at $90^{\circ}$ and $70^{\circ}$ angles respectively.

Figure 2 shows the needles position confirmed into the anterior sacral foramen in the 3D-computed tomography image, sagittal view).

When the needle is inserted deeply, the operator may feel slippery at first, and then a little sticky, but without resistance. At the same time, the patient may feel sore or a heavy feeling, radiating to the anus or perineum, or pelvis. There is no severe sharp pain. This technique is similar to implanting electrodes into the sacral foramen of the SNM procedure. 


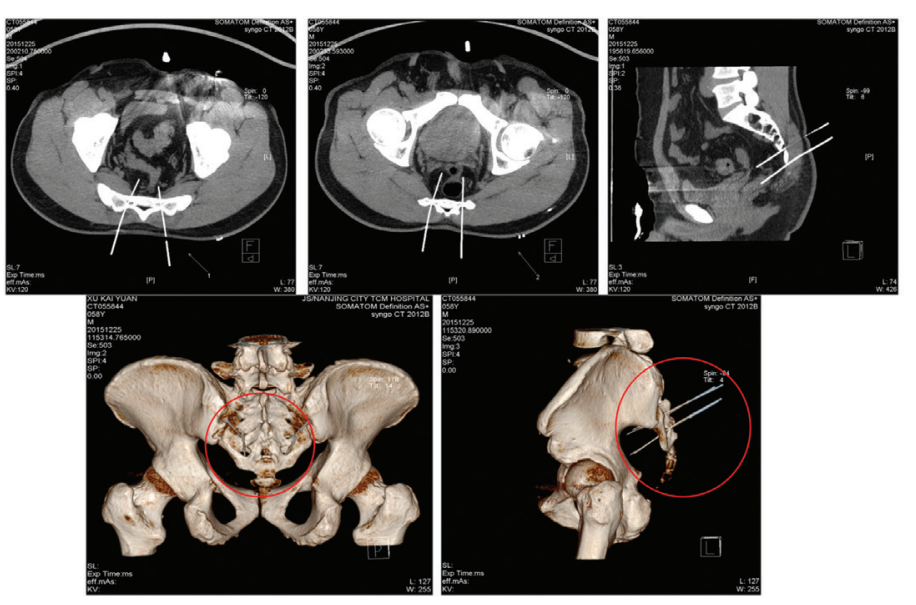

Figure 2. The needle position confirmed into the anterior sacral foramen in 3D-CT image, cross-section and sagittal view CT: Computed tomography
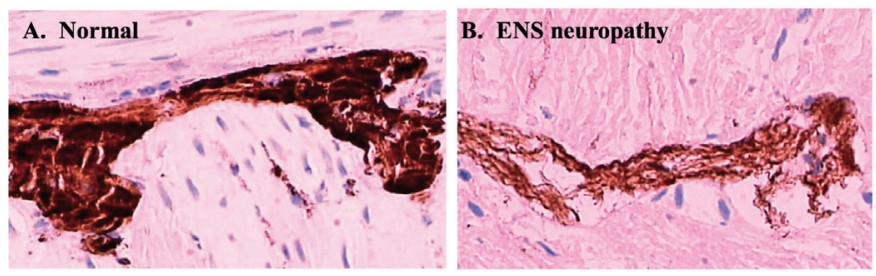

C. ENS neuropathy sham treatment
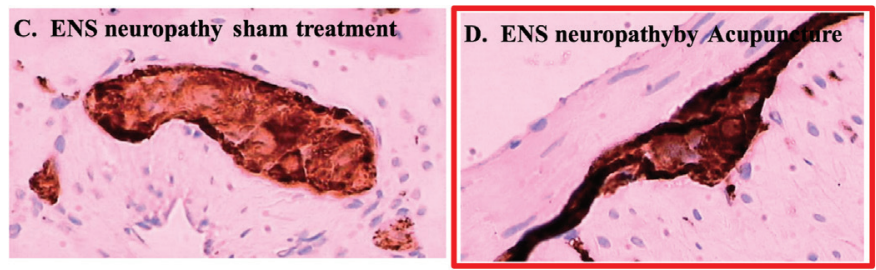

Figure 3. Protein gene product 9.5 (PGP9.5) positive expression in rat intestinal myenteric plexus $(\times 400)$

ENS: Enteric nervous system

\section{Appropriate electronic stimulation parameter ${ }^{28}$}

Electro-acupuncture is a method of regulating nerves or muscles function. That is to say, in terms of the functions of viscera, meridians, and qi, and blood in traditional Chinese medicine, the electro stimulator is connected to the needle handle of specific acupoints with a pair of positive and negative electrodes.

The electronic stimulator (Han's, LH 202 h, China) was connected on BL 33 (Zhong Liao) and BL 34 (Xia Liao) when in the prone position, while on ST 25 (Tian Shu) and SP 14 (Da Heng) when in the supine position for 30 minutes. By disperse-dense wave, 2/15 $\mathrm{Hz}$, and suitable intensity based on patients' tolerance, preferably with the skin around the acupoints shivering mildly without pain.

Electrical stimulation parameters were set from a single-center randomized controlled clinical trial. Randomized controls at $2 \mathrm{~Hz}, 15 \mathrm{~Hz}$, and $100 \mathrm{~Hz}$ were applied to patients with ODS to study their differences. The results showed that $15 \mathrm{~Hz}$ was the best for defecation, and $2 \mathrm{~Hz}$ and $15 \mathrm{~Hz}$ were better than $100 \mathrm{~Hz}$ for the stress and mental symptoms. And then a sparse-dense wave of $2 / 15 \mathrm{~Hz}$ was determined. ${ }^{29}$ It is different from other research parameters of patients with stress urinary incontinence (continuous wave of $50 \mathrm{~Hz}$ and a current intensity of 1 to $5 \mathrm{~mA}$ ). ${ }^{30}$

\section{Course and follow-up}

Routinely, the two sets of acupoints were used alternately, one set per day, with 20 sessions being a therapeutic course. Every session was for 30 minutes once daily. After a 4-week acupuncture session, it was found that dyssynergic defecation usually responded better than pelvic floor relaxation but if the treatment was prolonged to 8 weeks, the results reversed from this observation. So, some patients may need to prolong treatment courses if acupuncture responds slow.

Generally, the effects of acupuncture include the latent period, ascending period, peak period, and decline period. It may vary according to indications, stimulation parameters, and patient individuals. Some effects have a short latency and fast response, which is called rapid attack (neural reaction); On the contrary, it is called slow (e.g., humoral reaction).

Some patients may have resistance reaction, which means that there will be a good and rapid response during the acupuncture, and then there will be no result when given the same stimulus. If this is the case, it will be released after the end interval of 1 or 2 weeks, and it will be restored to its original effect or better.

Patients were given follow-up telephone calls at one and three months after the conclusion of treatment as scheduled by a research fellow. The follow-up data requested including Wexner Constipation Scoring System, ${ }^{31}$ stool consistency, awareness to defecate, and PAC-QOL in the research..$^{32}$ Other overlapping symptom responses may require other questionnaires.

Follow-up time is important and prolongs the treatment session intervals after the regular sessions, since function after acupuncture may deteriorate with time. Prolonging the treatment interval, ceasing gradually, is a way to obtain a longterm efficacy. If symptoms return, acupuncture still works when re-applied.

\section{Supporting evidence for acupuncture protocol in ODS}

Clinical evidence: ST 25 and ST 36 and deep needling for severe constipation. ${ }^{33}$

The author made a comprehensive exposition of the core acupoints in the above-mentioned ODS treatment, with emphasis on the functions and deep needling techniques of acupoints ST 25 and ST 36. This important result has been confirmed by a 
large paper entitled "Acupuncture Treatment of Chronic Severe Functional Constipation: Randomized Trial" published in the Western mainstream core journal "Annals of Internal Medicine" in the United States (US).

This multi-center trial had 1,075 participants from 13 hospitals, which showed that during the 8-week treatment period, electronic acupuncture can alleviate the symptoms of patients with chronic severe constipation and improve their quality of life; These effects persisted throughout the 12-week follow up.

\section{Experimental evidence: ST 25 and ST 36 repair the ENS ${ }^{34}$}

In the experimental scientific research, by comparing the expression of protein markers Protein Gene Product 9.5 (PGP 9.5) in normal group, Enteric nervous system (ENS) neuropathy group, ENS neuropathy sham treatment group, and ENS neuropathy acupuncture group, the author confirmed that acupoints ST 25 and ST 36 were beneficial to the recovery of intestinal function in ENS neuropathy model rats. ${ }^{33}$

The expression of PGP 9.5 was a characteristic marker for evaluating the function of intestinal ganglia. Figure 3 shows evidence of histopathological results.

Clinical evidence: sacral nerve modulation by acupuncture for pelvic floor dysfunction ${ }^{30}$

The author made a comprehensive exposition of the core acupoints in the above-mentioned ODS treatment, with emphasis on the functions and deep needling techniques of acupoints $\mathrm{BL}$ $33 \&$ BL 34. This important result has been confirmed by a large paper entitled "Effect of Electroacupuncture on Urinary Leakage Among Women with Stress Urinary Incontinence a Randomized Clinical Trial" published in the Western mainstream core journal "JAMA" in the US.

This multi-center, randomized, participant blind, and sham electroacupuncture controlled clinical study was carried out in 12 hospitals in China, and 504 female patients with stress urinary incontinence were recruited. Participants in the electroacupuncture group received acupuncture at bilateral $\mathrm{BL}$ 33 (Zhong Liao) and BL 35 (Hui Yang) with $50 \mathrm{~Hz}$ continuous-wave stimulation for 30 minutes. During the 6-week of 18 sessions treatment period, electroacupuncture involving the lumbosacral region showed a greater reduction in leakage of urine than sham electroacupuncture. The effect lasted 24 weeks after treatment.

According to this study, the emphasis is placed on bilateral BL 33 and deep needling technique (needle diameter is $0.30 \times 75 \mathrm{~mm}$ ), which is almost the same technique used by the author in ODS treatment.

\section{DISCUSSION}

\section{Potential pathophysiological mechanisms}

Acupuncture treatment for ODS is explained by two theories, the traditional meridian theory, and the modern nerveelectrophysiology theory.

From the neuroanatomy point of view, human defecation function is dominated by the CNS, ANS, and ENS. ANS innervation comes from C6 to T2 and S2 to S4 segments of the spinal cord and controls gastrointestinal function. It has been suggested that acupuncture could influence the visceral sensory system by stimulating the somatic sensory system. A series of investigations undertaken on somatoautonomic reflexes have provided good evidence of the importance of cutaneous input in autonomic control of Gl motility. ${ }^{22}$ The ENS takes its input from the intestine's myenteric plexus and Cajal cells, while CNS from the brain-gut axis. Stimulation of the corresponding points on the head (GV20) and back (BL23, BL25, and BL33-34, from the $3^{\text {rd }}$ to $4^{\text {th }}$ sacral foramen respectively) and abdomen (ST25 SP14 SP15 CV4 and (V6) will modulate the intestinal function.

It has been proven that stimulation of the sacral nerve can promote colonic motility, and improve pelvic floor sensation., ${ }^{4,21}$ These neurophysiological findings influenced our selection of acupoints for a clinical study. ${ }^{35,36}$ In our 90 cases of research on chronic functional constipation, over $90 \%$ of patients have sleeping dysfunction. We found that the acupoint GV20 can improve insomnia quickly, and has an indirect effect on bowel movement.

\section{CONCLUSION}

Acupuncture treatment has no requirements for ODS candidates, and it will react quickly compared to the pelvic floor biofeedback therapy. This is a minimally invasive, cost-effective, and flexible method compared to the SNM implantation procedure. Acupuncture is a multi-target holistic therapy, but it still has limitations for pelvic floor dysfunction. For different subset individuals, it is necessary to optimize the treatment scheme or may integrate other modalities.

In addition, acupuncture is not only a physical stimulus to the human body but also a face-to-face communication process between doctors and patients in the daily treatment, which plays the role of psychological counselling. Besides, acupuncture itself can improve mental and sleeping disorders through neural regulation.

Acupuncture has important potential for treatment and to be integrated into Western Medicine. It is an evidence- 
based contribution from the treatment of patients, and it is a combination of systematic "Tao" and individualized "technique". Enthusiasm for research is growing, and the cooperation of multidisciplinary teams will speed up the process of integrating acupuncture into conventional western medicine in near future.

\section{Contributions}

Concept: S.D., Y.D., L.W., H.Z., X.J., Design: S.D., Y.D., L.W., Data Collection or Processing: S.D., H.Z., X.J., Analysis or Interpretation: S.D., L.W., Literature Search: S.D., X.J., Writing: S.D., L.W., H.Z.

\section{Ethics}

Ethics Committee Approval: Not necessary for it is included several researches published data.

Informed Consent: Not necessary for it is included several researches published data.

Peer-review: Externally peer-reviewed.

\section{DISCLOSURES}

Conflict of Interest: No conflict of interest was declared by the authors.

Financial Disclosure: The authors declared that this study received no financial support.

\section{REFERENCES}

1. Podzemny V, Pescatori LC, Pescatori M. Management of obstructed defecation. World J Gastroenterol 2015; 21: 1053-60.

2. Paquette IM, Varma M, Ternent C, Melton-Meaux G, Rafferty JF, Feingold D, Steele SR. The American Society of Colon and Rectal Surgeons' Clinical Practice Guideline for the Evaluation and Management of Constipation. Dis Colon Rectum 2016; 59: 479-92.

3. Rao SS, Benninga MA, Bharucha AE, Chiarioni G, Di Lorenzo C, Whitehead WE. ANMS-ESNM position paper and consensus guidelines on biofeedback therapy for anorectal disorders. Neurogastroenterol Motil 2015; 27: 594-609.

4. Pilkington SA, Emmett C, Knowles CH, Mason J, Yiannakou Y; NIHR CapaCiTY working group; Pelvic floor Society. Surgery for constipation: systematic review and practice recommendations: Results V: Sacral Nerve Stimulation. Colorectal Dis 2017; 19: 92-100.

5. S Ding, $Y$ Ding, et al. Acupuncture in the treatment of chronic constipation: 31 case reports. [Article in Chinese] Zhong Hua Zhong Yi Yao Xue Kan 2008;26:434-6.

6. Jin X, Ding Y], Wang LL, et al. [Clinical study on acupuncture for treatment of chronic functional constipation]. Zhongguo Zhen Jiu 2010; 30: 97-101.

7. Zhou HF, Ding SQ, Ding Y], et al. [Observation on effect characteristics of electroacupuncture for different types of functional constipation]. Zhongguo Zhen Jiu 2014; 34: 435-8.
8. Ding S, Jin X, Ding Y, Wang L, Zhou H. Acupuncture in the treatment of chronic functional constipation[Chapter]. Complementary Therapies for the Body, Mind, and Soul. 2015 Sep 2nd:21.

9. Petros PE, Woodman PJ. The Integral Theory of continence. Int Urogynecol J Pelvic Floor Dysfunct 2008; 19: 35-40.

10. Davis K, Kumar D. Posterior pelvic floor compartment disorders. Best Pract Res Clin Obstet Gynaecol 2005; 19: 941-58.

11. Krogh K, Christensen P. Neurogenic colorectal and pelvic floor dysfunction. Best Pract Res Clin Gastroenterol 2009; 23: 531-43.

12. Sakakibara R, Uchiyama T, Yamanishi T, Shirai K, Hattori T. Bladder and bowel dysfunction in Parkinson's disease. J Neural Transm (Vienna) 2008; 115: 443-60.

13. Rao SS, Seaton K, Miller MJ, et al. Psychological profiles and quality of life differ between patients with dyssynergia and those with slow transit constipation. J Psychosom Res 2007; 63: 441-9.

14. Altomare DF, Spazzafumo L, Rinaldi M, Dodi G, Ghiselli R, Piloni $V$. Set-up and statistical validation of a new scoring system for obstructed defaecation syndrome. Colorectal Dis 2008; 10: 84-8.

15. Tantiphlachiva K, Rao P, Attaluri A, Rao SS. Digital rectal examination is a useful tool for identifying patients with dyssynergia. Clin Gastroenterol Hepatol 2010; 8: 955-60.

16. Brusciano L, Limongelli P, Pescatori M, et al. Ultrasonographic patterns in patients with obstructed defaecation. Int J Colorectal Dis 2007; 22: 969-77.

17. Piloni V, Tosi P, Vernelli M. MR-defecography in obstructed defecation syndrome (ODS): technique, diagnostic criteria and grading. Tech Coloproctol 2013; 17: 501-10.

18. Rao SS, Meduri K. What is necessary to diagnose constipation? Best Pract Res Clin Gastroenterol 2011; 25: 127-40.

19. Dumoulin C, Hay-Smith J, Frawley H, et al. 2014 consensus statement on improving pelvic floor muscle training adherence: International Continence Society 2011 State-of-the-Science Seminar. Neurourol Urodyn 2015; 34: 600-5.

20. Pedraza R, Nieto J, Ibarra S, Haas EM. Pelvic muscle rehabilitation: a standardized protocol for pelvic floor dysfunction. Adv Urol 2014; 2014: 487436.

21. Carrington EV, Evers J, Grossi U, et al. A systematic review of sacral nerve stimulation mechanisms in the treatment of fecal incontinence and constipation. Neurogastroenterol Motil 2014; 26 : 1222-37.

22. Takahashi T. Acupuncture for functional gastrointestinal disorders. J Gastroenterol 2006; 41: 408-17.

23. Kaptchuk TJ. Acupuncture: theory, efficacy, and practice. Ann Intern Med 2002; 136: 374-83.

24. Yang JW, Li QQ, Li F, Fu QN, Zeng XH, Liu CZ. The holistic effects of acupuncture treatment. Evid Based Complement Alternat Med 2014; 2014: 739708. 
25. Ding S, Jin X, Ding Y, Wang L, Zhou H. Acupuncture in Treatment of Chronic Functional Constipation. World Journal of AcupunctureMoxibustion 2011; 21

26. Gu, X Wan, L Wang. Clinical study on the treatment of functional constipation with shallow and deep acupuncture at Tianshu (ST 25) [Article in Chinese]. NJUCM 2010; 26: 415-7.

27. Wang L, Jin X. Re-understanding of the Ba-liao acupoints. [Article in Chinese].Journal of Nanjing University of Chinese Medicine. 2014; 30: 4-7.

28. Liu N.Clinical observation of electroacupuncture frequencies on obstructive defecation syndrome. [Dissertation in Chinese]. Nanjing University of traditional Chinese medicine, 2012

29. Zhou HF, Ding SQ, Ding Y], et al. [Observation on effect characteristics of electroacupuncture for different types of functional constipation]. Zhongguo Zhen Jiu 2014; 34: 435-8.

30. Liu Z, Liu Y, Xu H, et al. Effect of Electroacupuncture on Urinary Leakage Among Women With Stress Urinary Incontinence: A Randomized Clinical Trial. JAMA 2017; 317: 2493-501.

31. Agachan F, Chen T, Pfeifer J, Reissman P, Wexner SD. A constipation scoring system to simplify evaluation and management of constipated patients. Dis Colon Rectum 1996; 39: 681-5.
32. Marquis P, De La Loge C, Dubois D, McDermott A, Chassany 0 . Development and validation of the Patient Assessment of Constipation Quality of Life questionnaire. Scand J Gastroenterol 2005; 40: 540-51.

33. Liu Z, Yan S, Wu J, et al. Acupuncture for Chronic Severe Functional Constipation: A Randomized Trial. Ann Intern Med 2016; 165: 7619 .

34. Ding S, Ding Y, Ji X, et al. Effects of acupuncture on PGP9. 5 expressions in constipation model rats. World Chinese Journal of Digestology 2009; 17: 2184-7.

35. Shen EY, Chen FJ, Chen YY, Lin MF. Locating the Acupoint Baihui (GV20) Beneath the Cerebral Cortex with MRI Reconstructed 3D Neuroimages. Evid Based Complement Alternat Med 2011; 2011: 362494.

36. Kalavapalli R, Singareddy R. Role of acupuncture in the treatment of insomnia: a comprehensive review. Complement Ther Clin Pract 2007; 13: 184-93. 